\title{
Role of Maxillofacial Radiology and Imaging in the diagnosis and Treatment of Osteomyelitis of the Jaws
}

\author{
Deepak Gupta* \\ Department of Oral Medicine and Radiology, M.M. College of Dental Sciences and Research, Mullana, Ambala, Haryana, India
}

Received: June 27, 2015; Accepted: July 06, 2015; Published: July 16, 2015

*Corresponding author: Dr. Deepak Gupta, Department of Oral Medicine and Radiology, M.M. College of Dental Sciences and Research, Mullana, Ambala, Haryana, India, Tel: +91-9896671281; E-mail: drdeepak_26@rediffmail.com

Osteomyelitis is an inflammation of the bone due to infection caused by pathogenic microorganisms [1]. The disease process is empirically considered as alveolar osteitis when just alveolar bone is affected while the process is considered to be osteomyelitis when basal bone of the jaws is involved [2].

Radiological and Imaging techniques play a key role in the early diagnosis and follow up of inflammatory conditions of the skeleton [1-10]. The importance of radiology and imaging in the cases of Osteomyelitis of the jaws is well documented in the literature. Worth and Stoneman stated that imaging of osteomyelitis is accomplished by conventional radiography, supplemented as needed by Computed Tomography (CT) [6]. Yoshiura et al. recommended the use of CT to know the extension of the lesion. Further, Alabadi et al. stated the role of Magnetic Resonance Imaging (MRI), and radionucleide bone scanning in addition to conventional radiography and CT [4-6].

Radiographically, the differentiation between alveolar osteitis and osteomyelitis can be made by the location of the affected site. However, as alveolar bone also contains bone marrow, localized alveolar osteitis might be histopathologically diagnosed as osteomyelitis. Researchers have stressed crucially on the early diagnosis and adequate treatment for osteomyelitis failing which, acute osteomyelitis may progress to subacute or chronic stage. Henceforth, Imaging techniques play a key role in the early diagnosis and follow up of inflammatory conditions of the skeleton $[2,3]$.

Imaging of osteomyelitis is accomplished by conventional radiography, supplemented as needed by Computed Tomography (CT), Magnetic Resonance Imaging (MRI), and Radionucleide Bone Scanning to know the extension of the lesion. The significance of radiological evaluation is twofold a) To differentiate osteomyelitis from other conditions that show similar signs and symptoms and b) To check the progress of the disease and its response to the treatment $[2,6]$.

Adequate and rational application of radiology and imaging is directed to recognize, delineate and confirm a local source and extent of infection of the lower or upper jaw [2-4]. Further it also helps to recognize whether it is soft tissue or hard tissue infection and helps to differentially diagnose the lesion from similar lesions [5-9]. Sometimes the clinical presentation of chronic suppurative osteomyelitis of the jaws will look alike squamous carcinoma of the jaws. At that time it is highly crucial to differentially diagnose the lesion [8].

The first sign of osteomyelitis is loss of the trabecular structure of bone resulting in a focal area of radiolucency commonly related to an empty tooth socket or a diseased tooth. Initial radiographic indicators may be a widened periodontal ligament space or a defect of the lamina dura. Destruction of bone initially proceeds within cancellous bone. The cortical plate is secondarily involved by progressive bone resorption and increasing pressure exerted by the inflammation. Additional early signs are erosion of the endosteal contour of the basal mandibular cortical bone or in the upper jaw effacement of the contour of the alveolar maxillary recess. Plain films, even though initially frequently negative for osteomyelitis are able to display a potential Odontogenic source of infection [1-3].

Sequestra and periosteal bone formation serve as radiological "indicators" in the advanced stage of acute osteomyelitis and thus play a significant role in arriving at the diagnosis. Periosteal reactions may affect all nonalveolar borders of the mandible $[3,4]$. A periosteal reaction related to the maxilla is exceedingly rare. On conventional radiographs periosteal reactions consist of a predominantly single layered, linear radiopacity separated by a lucent line from the mandibular cortical bone. Related to the ease with which the periosteum is stripped from the mandibular cortex and the increased osteogenic potential, the periosteal reaction is more pronounced in young patients. The elevated periosteum lays down bone to form the "involucrum" that partially or completely surrounds the bone $[3,4]$. When occurring along the inferior aspect of the mandible periosteal new bone formation is best visualized on lateral oblique or panoramic radiographs $[2,3]$. A higher incidence of periosteal reaction on CT is noted when comparing conventional films and CT. This holds true for 
osseous fistulae as well. Consequently, with the exception of the panoramic view most of the plain films have been abandoned in favour of early application of a CT or MRI examination [2,3].

Ultrasonography can also aid in the diagnosis of osteomyelitis if fluid is present in direct contact with the bone, without intervening soft tissues. Other ultrasound findings consistent with osteomyelitis include elevation of the periosteum by more than $2 \mathrm{~mm}$ and thickening of the periosteum. Ultrasound may also improve the yield from fine needle biopsies as aspiration of the swelling can be planned guided by pus found in ultrasonography [1-3].

Henceforth there is an unmet need to screen the suspected patients of Osteomyelitis with pertinent imaging modality for early diagnosis, prompt treatment and better prognosis.

\section{References}

1. Sheikh S, Pallagatti S, Gupta D, Mittal A. Tuberculous osteomyelitis of mandibular condyle: a diagnostic Dilemma. Dentomaxillofac Radiol. 2012; 41(2): 169-174. doi: 10.1259/dmfr/56238546.

2. Topazian RG. Osteomyelitis of the jaws. In: oral and maxillofacial infections. 2002. p. 214-239
3. Baltensperger MM, Eyrich GK. Osteomyelitis of jaws. Springer; 2009.

4. Aliabadi P, Nikpoor N. Imaging Osteomyelitis. Arthritis and Rheumstism. 1994; 37(5): 617-622.

5. Yoshiura K1, Hijiya T, Ariji E, Sa'do B, Nakayama E, Higuchi Y, et al. Radiographic patterens of osteomyelitis in the mandible: Plain film/ CT correlation. Oral Surg Oral Med Oral Pathol. 1994; 78(1): 116-24.

6. Worth HM, Stoneman DM. Osteomyelitis, malignant disease, and fibrous dysplasia: some radiologic similarities and differences. Dent Radiogr Photogr 1977; 50 (1): 1-8, 12-5.

7. Taori KB, Solanke R, Mahajan SM, Rangankar V, Saini T. CT Evaluation of mandibular osteomyelitis. Ind J Radiol Imag 2005; 15 (4): 447-451.

8. Sheikh S, Pallagatti S, Aggarwal A, Gupta D, Puri N, Mittal A. Osteosarcoma of maxilla: A case report. J Clin Exp Dent. 2010; 2(3): e117-20

9. Gupta D, Sheikh S, Pallagatti S, Aggarwal A, Singh R, Mittal A. Osteomyelitis of the mandible mimicking fibrous dysplasia: A radiographic controversy. Clinical Dentistry 2013; 7(3): 20-25.

10. Gupta D. Oro-Maxillofacial Radiology and Imaging: An Indispensible Dental Speciality. The Open Dentistry Journal, 2015, 9, (Suppl 2: M1) 260-262. 